Nonlin. Processes Geophys., 25, 441-455, 2018

https://doi.org/10.5194/npg-25-441-2018

(C) Author(s) 2018. This work is distributed under

the Creative Commons Attribution 4.0 License.

\title{
The evolution of mode- 2 internal solitary waves modulated by background shear currents
}

\author{
Peiwen Zhang ${ }^{1,3,4}$, Zhenhua $\mathrm{Xu}^{1,2,3,4}$, Qun $\mathrm{Li}^{5}$, Baoshu Yin ${ }^{1,2,3,4}$, Yijun $\mathrm{Hou}^{1,2,3,4}$, and Antony K. Liu ${ }^{6}$ \\ ${ }^{1}$ Key Laboratory of Ocean Circulation and Waves, Institute of Oceanology, Chinese Academy of Sciences, \\ Qingdao, 266071, China \\ ${ }^{2}$ Qingdao National Laboratory for Marine Science and Technology, Qingdao, 266071, China \\ ${ }^{3}$ University of the Chinese Academy of Sciences, Beijing, 100049, China \\ ${ }^{4}$ Center for Ocean Mega-Science, Chinese Academy of Sciences, Qingdao, 266071, China \\ ${ }^{5}$ Polar Research Institute of China, Shanghai, 200136, China \\ ${ }^{6}$ Ocean University of China, Qingdao, 266100, China
}

Correspondence: Zhenhua Xu (xuzhenhua@qdio.ac.cn) and Qun Li (liqun@pric.org.cn)

Received: 25 December 2017 - Discussion started: 15 January 2018

Revised: 29 May 2018 - Accepted: 4 June 2018 - Published: 25 June 2018

\begin{abstract}
The evolution of mode-2 internal solitary waves (ISWs) modulated by background shear currents was investigated numerically. The mode-2 ISW was generated by the "lock-release" method, and the background shear current was initialized after the mode- 2 ISW became stable. Five sets of experiments were conducted to assess the sensitivity of the modulation process to the direction, polarity, magnitude, shear layer thickness and offset extent of the background shear current. Three distinctly different shear-induced waves were identified as a forward-propagating long wave, oscillating tail and amplitude-modulated wave packet in the presence of a shear current. The amplitudes of the forwardpropagating long wave and the amplitude-modulated wave packet are proportional to the magnitude of the shear but inversely proportional to the thickness of the shear layer, as well as the energy loss of the mode- 2 ISW during modulation. The oscillating tail and amplitude-modulated wave packet show symmetric variation when the background shear current is offset upward or downward, while the forwardpropagating long wave was insensitive to it. For comparison, one control experiment was configured according to the observations of Shroyer et al. (2010); in the first 30 periods, $\sim 36 \%$ of total energy was lost at an average rate of $9 \mathrm{~W} \mathrm{~m}^{-1}$ in the presence of the shear current; it would deplete the energy of initial mode- 2 ISWs in $\sim 4.5 \mathrm{~h}$, corresponding to a propagation distance of $\sim 5 \mathrm{~km}$, which is consistent with in situ data.
\end{abstract}

\section{Introduction}

Internal solitary waves (ISWs) are commonly observed in stratified oceans, especially in coastal and continental shelf regions (Grimshaw et al., 2010; Helfrich and Melville, 2006; Lamb, 2014). While mode-1 ISWs are frequently observed by in situ observations (Farmer et al., 2009; Klymak and Moum, 2003; Moum et al., 2006) and by remote sensing (Liu et al., 1998, 2004; Zhao et al., 2004; Zhao and Alford, 2006), higher modes are relatively rarely captured (Jackson et al., 2013). Even so, with the improvement of observation, mode-2 ISWs have been reported recently (Liu et al., 2013; Shroyer et al., 2010; Yang et al., 2009, 2010). Most previously reported mode-2 ISWs were categorized as convex types, which have the potential to transport mass (Brandt and Shipley, 2014; Deepwell and Stastna, 2016; Salloum et al., 2012). In contrast, concave mode-2 ISWs are seldom observed because the stratification with a thick middle layer is rare (Yang et al., 2010)

The majority of studies of mode-2 ISWs aimed at interpreting their generation mechanisms under different conditions (Helfrich and Melville, 1986; Huttemann and Hutter, 2001; Stastna and Peltier, 2005; Vlasenko et al., 2010). Under most circumstances, mode-2 ISWs show a specific phenomenon of a "short-lived" nature (Ramp et al., 2012; Terletska et al., 2016; Yuan et al., 2018). Ramp et al. (2012) concluded that mode-2 ISWs around the HengChun Ridge would dissipate in $8.9 \mathrm{~h}$, suggesting mode-2 
ISWs are highly dissipative when travelling around rough topographical features. Terletska et al. (2016) showed the decaying of mode-2 ISWs was induced by step-like topography. The forward-propagating long waves, breather-like internal waves (BLIWs) and oscillating tail were generated during the adjustment process. Yuan et al. (2018) observed the existence of a long mode-1 wave ahead of mode-2 ISWs during the evolution of mode-2 ISWs over variable topography and found that this process cannot be characterized by Kortewegde Vries (KdV) theory. The authors suggested using the MIT$\mathrm{gcm}$ model, which can solve all modes to investigate the integrated evolution process of mode-2 ISWs under variable background conditions.

The ephemeral phenomenon of mode-2 ISWs could also be induced by background shear currents. They are more common in the open ocean because they can be induced by baroclinic eddies, baroclinic tides, wind and mode-1 ISWs (Chen et al., 2011; Stastna et al., 2015; Wang et al., 1991; $\mathrm{Xu}$ et al., 2013, 2016). The evolution of mode-1 ISWs in background shear currents was extensively studied (Lamb, 2010; Grimshaw et al., 2007; Stastna and Lamb, 2002). Lamb (2010) investigated the energetics of mode-1 ISWs in a background shear current, providing some methods commonly used to calculate the energy under that circumstance. Stastna and Lamb (2002) considered the effects of background current on mode-1 ISWs and discussed the properties of ISWs during the breaking process. In comparison, few works on mode-2 ISWs in shear flow have been produced. Vlasenko et al. (2010) observed mode-2 ISWs followed by a short wavelength mode-1 oscillating tail in the Luzon Strait. Liu et al. (2013) investigated the generation and evolution of mode-2 ISWs in the South China Sea and concluded that the more dispersive mode-2 ISWs might not propagate, evolve and persist for a long time on the shelf.

Their works focused on the shoaling pycnocline and topography, which were suggested to cause ephemeral mode-2 ISWs, but the effects of background shear currents were neglected. Shroyer et al. (2010) was the sole one recording an integrated evolution process of mode-2 ISWs and found that leading mode- 2 waves quickly deformed and developed a tail of short, small-amplitude mode- 1 waves in the presence of a background shear current. Therefore, the authors speculated that the background shear currents could produce instabilities and lead to the adjustment of ISWs, and they also concluded that the wave-localized turbulence dissipation was comparable with that induced by mode-1 ISWs. Motivated by the results of Shroyer et al. (2010), we numerically investigated the effect of shear currents on evolution processes of mode2 ISWs. To reveal the sensitivity of the evolution of mode-2 ISWs to variable parameters of background shear currents, we introduced five sets of experiments (21 experiments in total) to generalize our research, including the magnitude, thickness of the shear layer, polarity, direction and offset (centre of the shear layer relative to the centre of the pycnocline) of the background shear current. These conditions are common in the oceans; for example, the internal tidal wave could be symmetric around the pycnocline (Duda et al., 2004), but in the shelf-slope area, the surface-intensified flow driven by wind could deviate for a distance from the centre of the pycnocline (Van de Boon, 2011).

The remainder of the paper is organized as follows: the numerical model configurations and background conditions are detailed in Sect. 2. In Sect. 3, the modulation process of a mode-2 ISW in background shear currents and its sensitivity to varied background shear currents are presented and assessed. The decaying of a mode-2 ISWs' energy and the effect of a varied background shear current on it are analysed and summarized in Sect. 4. In Sect. 5, details of mode-2 ISW evolution and characteristics of shear-induced waves are compared and discussed. Then, the results are summarized in Sect. 6.

\section{Numerical model and background condition}

\subsection{Model configuration}

Our numerical simulations are based on the Massachusetts Institute of Technology general circulation model (MITgcm, Marshall et al., 1997). The nonhydrostatic capability of the MITgcm is turned on because ISWs represent a balance of nonlinearities and dispersions, with the latter derived from nonhydrostatic pressure.

A series of 2-D numerical simulations were performed to investigate the evolution of mode-2 ISWs in the presence of background shear currents. The experimental domain was $12.8 \mathrm{~km}$ long and $100 \mathrm{~m}$ deep. The horizontal and vertical resolutions were $2.5 \mathrm{~m}$ with 5120 grid points and $0.5 \mathrm{~m}$ with 200 grid points, respectively. The time step was $0.4 \mathrm{~s}$ in order to ensure that the Courant-Friedrichs-Lewy (CFL) condition was satisfied and the model was stable. The viscosity parameters were set to $10^{-3} \mathrm{~m}^{2} \mathrm{~s}^{-1}$ for the horizontal viscosity $v_{\mathrm{H}}$ and $10^{-4} \mathrm{~m}^{2} \mathrm{~s}^{-1}$ for the vertical viscosity $v_{\mathrm{v}}$ in the present study. The flux-limiting advection scheme for the tracers introduces numerical diffusivity which is needed for stability, so the explicit diffusivity was set to zero (Legg and Adcroft, 2003; Legg and Huijts, 2006; Legg and Klymak, 2008).

\subsection{Stratification and background conditions}

The choice of background density stratification in all experiments and the shear current in the control experiment (case O5) followed the field observation over the New Jersey Shelf (Shroyer et al., 2010). The background stratification and shear currents adopted the hyperbolic tangent function for smoothing, which can be written as follows:

$\rho(z)=\rho_{0}-\frac{\Delta \rho}{2} \tanh \left[\frac{\left(z+z_{0}\right)}{h}\right]$,

where $\rho_{0} \equiv\left(\rho_{1}+\rho_{2}\right) / 2$ is the mean density of the vertical water column and $\Delta \rho \equiv\left(\rho_{2}-\rho_{1}\right)$ is the difference in den- 
(a) Density

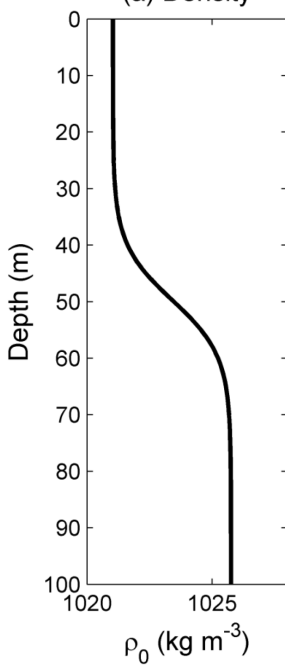

(b) Brunt-Vaisala frequency

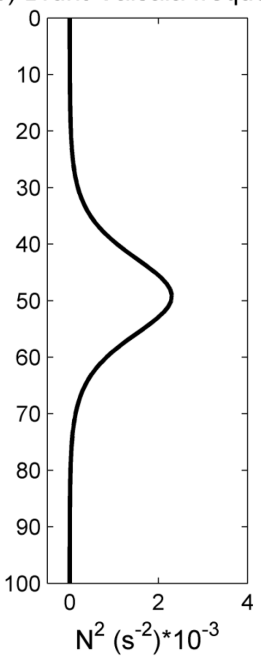

(c) Current

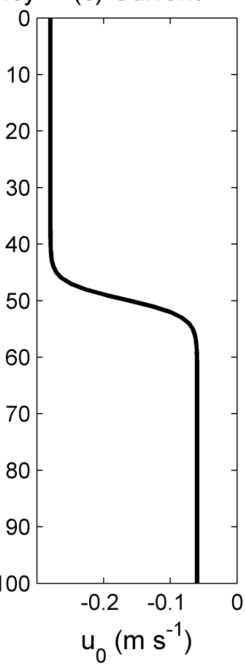

Figure 1. Vertical profiles of the (a) density, (b) buoyancy frequency and (c) background velocities in the control experiment (case O5).

sity between the upper layer $\rho_{1}\left(1022 \mathrm{~kg} \mathrm{~m}^{-3}\right)$ and bottom layer $\rho_{2}\left(1026 \mathrm{~kg} \mathrm{~m}^{-3}\right) . h$ is the pycnocline thickness and $z_{0}$ is the depth of the pycnocline centre. The function of the background shear current is given by

$U(z)=U_{0}-\frac{\Delta U}{2} \tanh \left[\frac{\left(z+D_{\mathrm{s}}\right)}{h_{\mathrm{s}}}\right]$,

where $U_{0} \equiv\left(U_{1}+U_{2}\right) / 2$ is the mean background velocity of the water column, $\Delta U \equiv\left(U_{2}-U_{1}\right)$ is the difference in the background horizontal velocity between the upper layer $U_{1}$ and bottom layer $U_{2}$, and $h_{\mathrm{s}}$ is the thickness of the shear layer.

In sensitivity experiments, the magnitude of the shear current is denoted by $U_{\mathrm{d}}$ and defined as $|\Delta U|$. This value was varied from $0.5 U_{\mathrm{d}}$ to $2.5 U_{\mathrm{d}}$ (here $U_{\mathrm{d}}=0.22 \mathrm{~m} \mathrm{~s}^{-1}$ ) in a sensitivity test. Similarly, the thickness of the shear layer was varied from $0.5 h_{\mathrm{s}}$ to $2.5 h_{\mathrm{s}}$ (here $h_{\mathrm{s}}=3 \mathrm{~m}$ ). In cases D1 and $\mathrm{P} 1$, opposing and polarity-reversal background shear currents were initialized for examination, respectively. We further introduced an asymmetry parameter $\Delta$ to investigate the evolution of mode-2 ISWs in offset background shear currents (Carpenter et al., 2010). The asymmetry parameter $\Delta$ is defined as follows:

$\Delta=\frac{D_{\mathrm{s}}-z_{0}}{h / 2}$,

where $D_{\mathrm{s}}$ denotes the depth of the shear centre and $h$ denotes the thickness of the pycnocline. $\Delta$ was varied from -2 to 2 (case O1 to case O9) to investigate the evolution of the mode-2 ISW in the offset background shear current. The vertical distributions of the background shear currents, density and buoyancy frequency in the control experiment are shown
Table 1. Summary of parameters of variable background shear currents. The depth and thickness of the pycnocline are denoted by $z_{0}$ and $h$. The thickness of the shear layer is denoted by $h_{\mathrm{s}}$, and the offset cases are indicated by asymmetry parameter $\Delta$. The magnitude of the shear current is denoted by $U_{\mathrm{d}}$, and $U_{0}$ is the mean background velocity of the water column. Por indicates the polarity of the background shear current, and "+" means a polarity-reversal shear current. The orientation of the background shear current is indicated by Ori, and "+" means an opposing shear current.

\begin{tabular}{lrrrrrrrr}
\hline Case & $\begin{array}{r}\mathbf{z}_{0} \\
(\mathrm{~m})\end{array}$ & $\begin{array}{r}h \\
(\mathrm{~m})\end{array}$ & $\begin{array}{r}h_{\mathbf{s}} \\
(\mathrm{m})\end{array}$ & $\Delta$ & $\begin{array}{r}U_{\mathbf{0}} \\
\left(\mathrm{m} \mathrm{s}^{-1}\right)\end{array}$ & $\begin{array}{r}U_{\mathbf{d}} \\
\left(\mathrm{m} \mathrm{s}^{-1}\right)\end{array}$ & Por & Ori \\
\hline O1 & 50 & 10 & 3 & -2 & 0.17 & 0.22 & $(-)$ & $(-)$ \\
O2 & 50 & 10 & 3 & -1.5 & 0.17 & 0.22 & $(-)$ & $(-)$ \\
O3 & 50 & 10 & 3 & -1 & 0.17 & 0.22 & $(-)$ & $(-)$ \\
O4 & 50 & 10 & 3 & -1.5 & 0.17 & 0.22 & $(-)$ & $(-)$ \\
O5 & 50 & 10 & 3 & 0 & 0.17 & 0.22 & $(-)$ & $(-)$ \\
O6 & 50 & 10 & 3 & 0.5 & 0.17 & 0.22 & $(-)$ & $(-)$ \\
O7 & 50 & 10 & 3 & 1 & 0.17 & 0.22 & $(-)$ & $(-)$ \\
O8 & 50 & 10 & 3 & 1.5 & 0.17 & 0.22 & $(-)$ & $(-)$ \\
O9 & 50 & 10 & 3 & 2 & 0.17 & 0.22 & $(-)$ & $(-)$ \\
\hline H1 & 50 & 10 & 1.5 & 0 & 0.17 & 0.22 & $(-)$ & $(-)$ \\
H2 (O5) & 50 & 10 & 3 & 0 & 0.17 & 0.22 & $(-)$ & $(-)$ \\
H3 & 50 & 10 & 4.5 & 0 & 0.17 & 0.22 & $(-)$ & $(-)$ \\
H4 & 50 & 10 & 6 & 0 & 0.17 & 0.22 & $(-)$ & $(-)$ \\
H5 & 50 & 10 & 7.5 & 0 & 0.17 & 0.22 & $(-)$ & $(-)$ \\
\hline U1 & 50 & 10 & 3 & 0 & 0.225 & 0.11 & $(-)$ & $(-)$ \\
U2 (O5) & 50 & 10 & 3 & 0 & 0.17 & 0.22 & $(-)$ & $(-)$ \\
U3 & 50 & 10 & 3 & 0 & 0.115 & 0.33 & $(-)$ & $(-)$ \\
U4 & 50 & 10 & 3 & 0 & 0.06 & 0.44 & $(-)$ & $(-)$ \\
U5 & 50 & 10 & 3 & 0 & 0.005 & 0.55 & $(-)$ & $(-)$ \\
\hline D1 & 50 & 10 & 3 & 0 & -0.17 & 0.22 & $(-)$ & $(+)$ \\
\hline P1 & 50 & 10 & 3 & 0 & 0.17 & 0.22 & $(+)$ & $(-)$ \\
\hline
\end{tabular}

in Fig. 1. Detailed configurations of the numerical simulation in the present study are given in Table 1 . In all cases, the Richardson numbers of the background current were estimated to be larger than 0.25 , indicating the stable state of the background environment.

\subsection{Model initialization}

A rank-ordered mode-2 ISW train was generated by the "lock-release" method without background current (Brandt and Shipley, 2014; Deepwell and Stastna, 2016; Olsthoorn et al., 2013; Stastna et al., 2015). Figure 2 demonstrates a schematic diagram of the initialization process and the configuration of model parameters. A mixed region was set to be symmetric around the centre line of the pycnocline at the right end, and its length $l_{\text {mix }}$ and height $h_{\text {mix }}$ were $375 \mathrm{~m}$ and $25 \mathrm{~m}$, respectively. The pycnocline was $10 \mathrm{~m}$ thick, and this dimension was indicated by $h$. The model was initialized at $t=0 \mathrm{~s}$, and the rank-ordered mode- 2 wave train emerged at $t=4000 \mathrm{~s}$ and propagated to the left of the domain; as shown in Fig. 2, the leading mode-2 wave was extracted at that time and then propagated for $2000 \mathrm{~s}$ until it stabilized. The amplitude $A$ of mode-2 ISWs was defined as the maximum dis- 


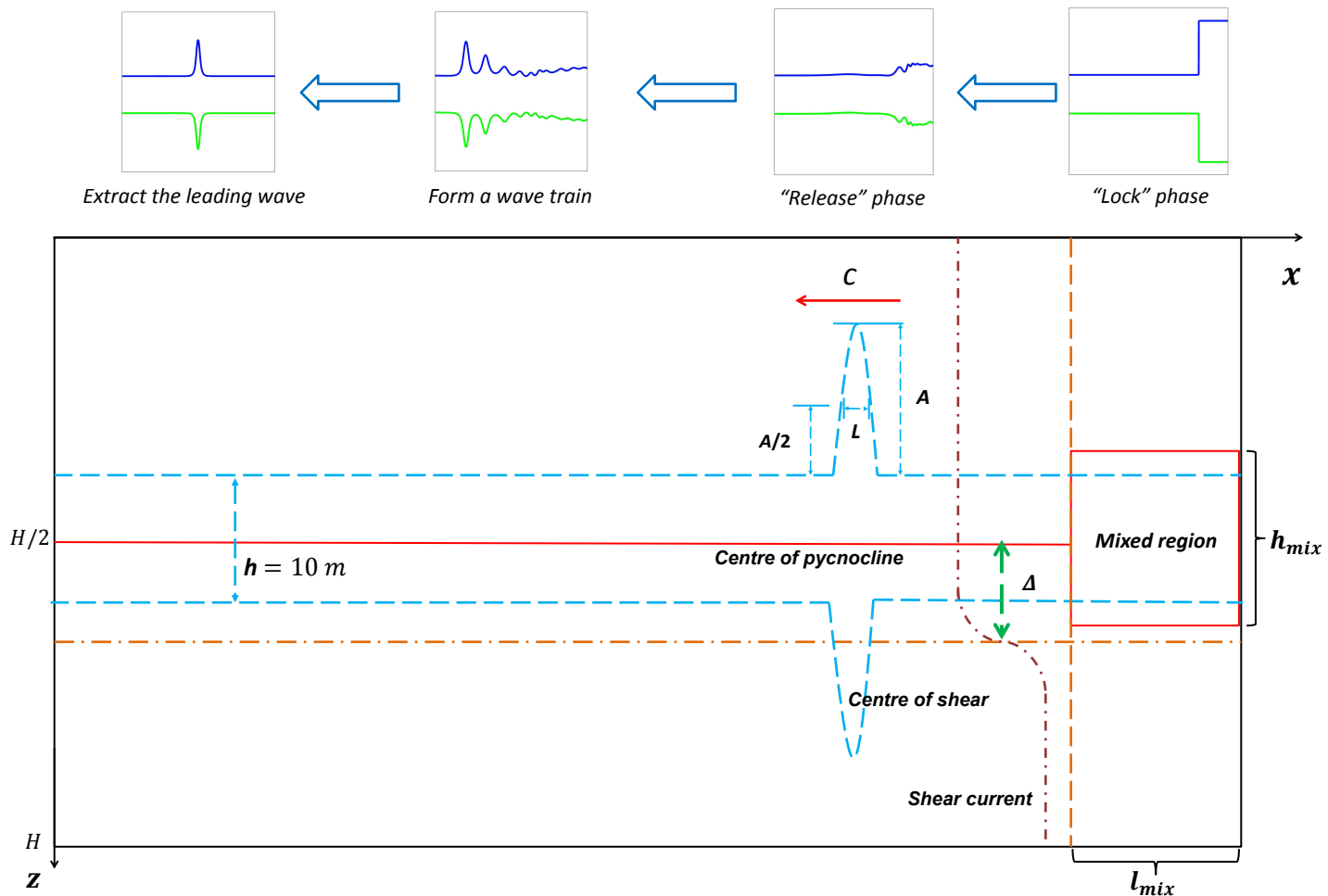

Figure 2. Schematic diagram of the model configuration and initialization, where $\Delta$ denotes the asymmetry parameter, $h$ denotes the thickness of the pycnocline, $c, A$, and $L$ indicate the propagation speed, amplitude, and wavelength of a mode- 2 ISW, $h_{\text {mix }}$ and $l_{\text {mix }}$ denote the height and length of the mixed region, and $H$ indicates the depth.
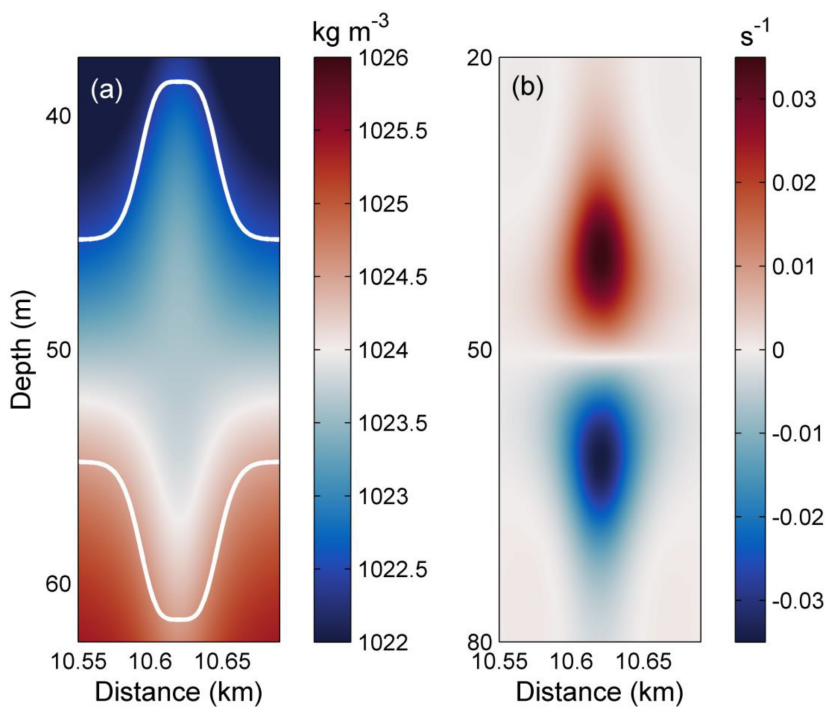

Figure 3. The characteristics of a single mode-2 ISW's (a) density field and (b) vorticity in the absence of a background shear current. The white lines in (a) demonstrate the theoretical solution of a mode-2 ISW in the KdV framework.

placement of the upper and lower isopycnals, which are equal in the initial state (Terletska et al., 2016). The wavelength $L$ was defined as the width of the wave at half of the amplitude of the mode-2 ISW in the initial state. At $6000 \mathrm{~s}$ after the initialization of the numerical model, the velocity field of the background shear current was superimposed on the model.

\section{Results}

\subsection{Characteristics of mode-2 ISWs}

The characteristics of an initial mode-2 ISW were compared with KdV theory (Grimshaw et al., 2010). The vorticity and density fields of the leading single wave of a mode-2 ISW in the absence of the background shear current are shown in Fig. 3, and the wave exhibits a notably symmetric structure. The ISW had an amplitude of $7 \mathrm{~m}$ and a wavelength of $62.5 \mathrm{~m}$ with a propagation speed at $\sim 0.31 \mathrm{~m} \mathrm{~s}^{-1}$, which was defined as $c$. It is slightly larger than the linear long-wave phase speed $c_{\mathrm{p}}\left(0.295 \mathrm{~m} \mathrm{~s}^{-1}\right)$ calculated by the Taylor-Goldstein equation (Vlasenko et al., 2010) and non-dimensionalized by long-wave phase speed as 1.05 . The typical timescale $T$ for mode- 2 ISWs is $200 \mathrm{~s}$, which was calculated by $L / c$. The modelled profile of the mode- 2 wave is consistent with the theoretical solution of the mode-2 ISW in the framework of the KdV equation (white lines in Fig. 3). 

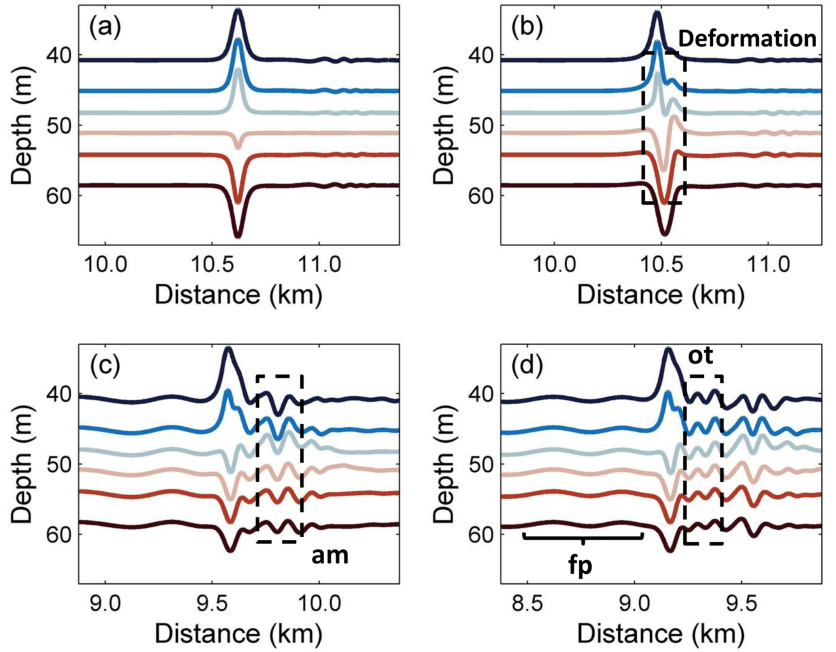

Figure 4. The evolution process of the mode- 2 ISW in case O5 ( $\Delta=0$ ) for the different times (a) $0 T$, (b) $1.2 T$, (c) $10 T$, and (d) $14 T$, where "am", "fp" and "ot" denote the amplitude-modulated wave packet, forward-propagating long wave and oscillating tail, respectively.

\subsection{The evolution of mode-2 ISWs in control experiments}

The evolution of a mode-2 ISW modulated by background shear current in the control experiment (case O5) is shown in Fig. 4, with its corresponding vorticity field shown in Fig. 5. In the initial state $(0 T)$, the mode- 2 ISW was symmetric about the pycnocline centre and the vorticities of the upper and lower parts counterbalance each other, demonstrating a dipole structure (Fig. 4a and Fig. 5a).

Then, at $1.2 T$ after the presence of a background shear current, as shown in Fig. 5b, the shear led to the deformation of the dipole, with the upper part being pushed forward. It also caused the asymmetrical distribution of the vorticity in the horizontal, which is associated with the generation of forward-propagating long waves and the amplitudemodulated wave packet (Fig. 4b), and the latter was defined as a pulsating wave packet (Clarke et al., 2000). The pulsating wave packet propagated with a steady-state envelope, inside which the waves oscillate freely with different amplitudes (Terletska et al., 2016). To the aft of the ISW, the shear induced by background currents led to the deformation of the vortex dipole, and an increasing complexity of the vorticity field implied an intensive adjustment occurred. As illustrated in Fig. 5c, the vorticity of the mode2 ISW is redistributed to adapt to the background condition at $10 T$. In this process, the vortex of the ISW shrank with the generation of an amplitude-modulated wave packet and a forward-propagating long wave. The amplitudes of shearinduced waves were defined as maximum isopycnal displacement (Stastna and Lamb, 2002). The forward-propagating

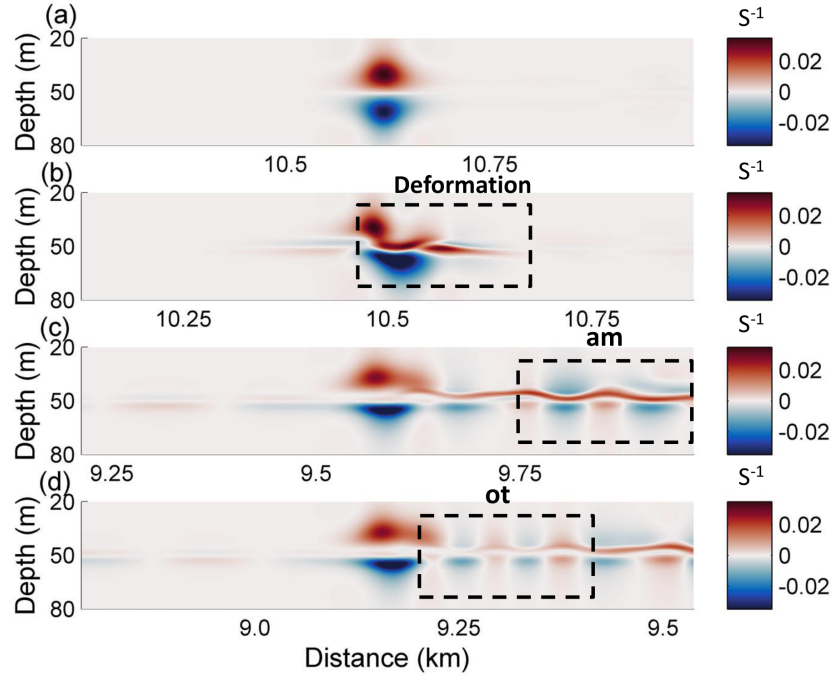

Figure 5. The evolution process of the vorticity field $\left(\mathrm{s}^{-1}\right)$ in case O5 $(\Delta=0)$ for the different times (a) $0 T$, (b) $1.2 T$, (c) $10 T$, and (d) $14 T$, where "am" and "ot" denote the amplitude-modulated wave packet and oscillating tail, respectively.

long wave and amplitude-modulated wave packet can be seen in Fig. $4 \mathrm{c}$ with amplitudes of 0.25 and $1.8 \mathrm{~m}$ respectively, and the latter was clearly observed at the rear of the mode-2 ISW.

The oscillating tail caused by shear was visible between the mode-2 ISW and the amplitude-modulated wave packet (Fig. 4d) at $14 T$, and it was a radiated mode-1 oscillatory disturbance trailing mode-2 ISW (Stamp and Jacka, 1995). A Hovmöller plot (Fig. 6) of horizontal velocity without the background shear current at the surface was plotted. The forward-propagating long wave, oscillating tail and amplitude-modulated wave packet were found to propagate persistently. The amplitude-modulated wave packet propagated independently from the ISW, indicating that it generated transiently. Based on the vorticity field (Fig. 5d) and the time-space varying nature (Fig. 6), the generation of the oscillating tail and the forward-propagating long wave was continuously sustained by the energy of the ISW with decreasing rate. Therefore, they have the potential to drain the energy from an ISW over a long timescale. The traces of a forward-propagating long-wave and amplitude-modulated wave packet appear at the same time (Fig. 6). The oscillating tail appears later than the amplitude-modulated wave packet, and is located between the mode- 2 ISW and amplitudemodulated wave packet (Fig. 6). Thus, the energy loss of the ISW caused by forward-propagating long waves occurs earlier than the oscillating tail.

\subsection{The evolution of mode-2 ISWs in offset background shear currents}

The influence of the offset background shear current on the modulation of a mode-2 ISW was investigated, and in offset 


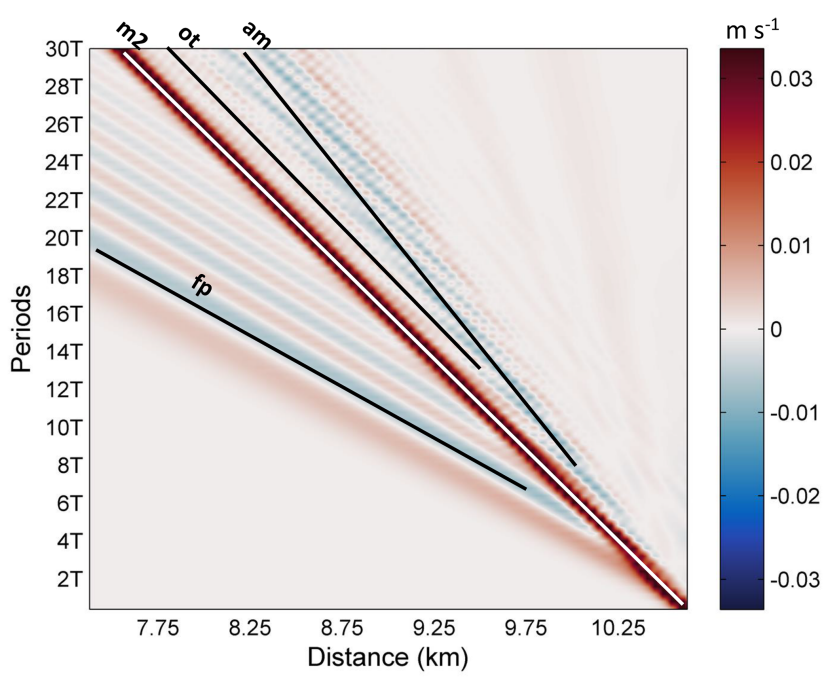

Figure 6. Hovmöller plot of horizontal velocity without the background shear current at the surface. The mode-2 ISW, forwardpropagating long wave, oscillating tail and amplitude-modulated wave packet are denoted by "m2", "fp", "ot" and "am", respectively.
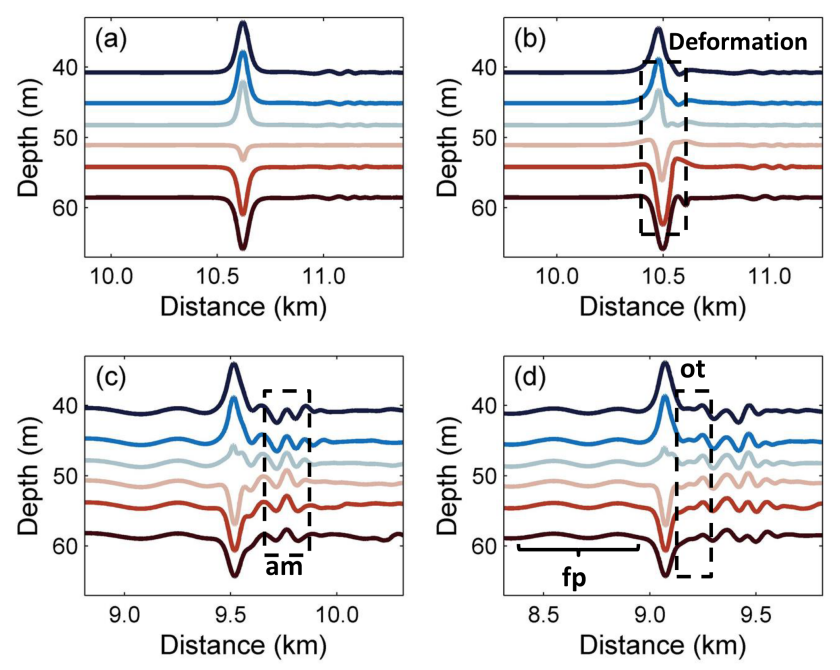

Figure 7. The evolution processes of the mode-2 ISW in case O9 $(\Delta=2.0)$ for the different times (a) $0 T$, (b) $1.2 T$, (c) $10 T$, and (d) $14 T$, where "am", "fp" and "ot" denote the amplitude-modulated wave packet, forward-propagating long wave and oscillating tail, respectively.

cases the shear current was set to deviate from the centre of the pycnocline with varied asymmetry parameters. We take case $09(\Delta=2)$ for a detailed examination in the following section. The evolution of the mode-2 ISW in case O9 and its corresponding vorticity field are shown in Fig. 7 and Fig. 8, respectively.

The deformation of the vortex dipole at $1.2 \mathrm{~T}$ after the initialization of a background shear current is illustrated in Fig. 8b. The shear vertically distorted the lower section of the

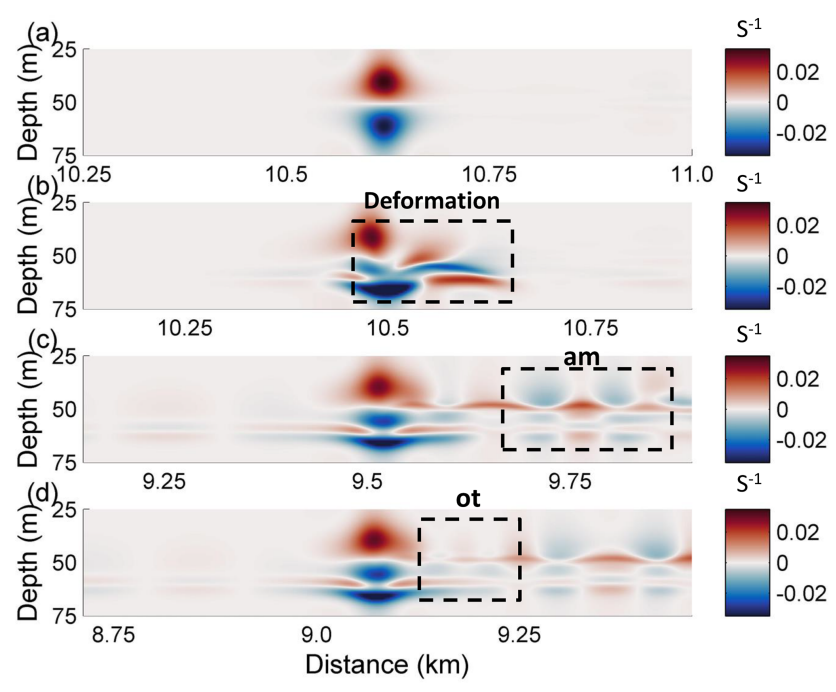

Figure 8. The evolution process of the vorticity field in case $\mathrm{O} 9$ ( $\Delta=0$ ) for the different times (a) $0 T$, (b) $1.2 T$, (c) $10 T$, and (d) $14 T$, where "am" and "ot" denote the amplitude-modulated wave packet and oscillating tail, respectively.

vortex and forced some of the vortices from the lower part of the dipole to penetrate the upper part. Redistribution of vorticity occurred both vertically and horizontally. The upper section was bifurcated such that the branch containing most of the vorticity intruded ahead, which related to the generation of a forward-propagating long wave in the same manner as that in the control experiment, as shown in Fig. 7b. The other branch moved backward to the aft of the ISW, corresponding to the generation of an amplitude-modulated wave packet. Given this modulation process, while the lower part of the dipole obviously shrank, the upper parts slowly reunited to restore the vertical balance.

The vorticity distribution was different from that of the control experiment at $10 T$ (Fig. 8c). The lower part of the vortex had two obvious cores, and they were separated by shear effect and jointly balanced with the upper section. The amplitude-modulated wave packet and forwardpropagating long waves are plotted (Fig. 7c). The amplitude of the wave packet was $1 \mathrm{~m}$, which was smaller than that of the control experiment. However, the amplitude of the forward-propagating long wave was still approximately $0.2 \mathrm{~m}$. In Fig. $7 \mathrm{~d}$, an oscillating tail with $0.25 \mathrm{~m}$ amplitude developed at the rear of the wave. It was sustained by the energy transferred from the ISW, as shown in Fig. 8d. When the shear current is offset in the upward direction $(\Delta<0)$, the asymmetry of the mode- 2 ISW during the modulation became clearer. The amplitudes of the oscillating tail and amplitude-modulated wave packet both decreased when the shear current was offset upward, showing a symmetric variation trend with respect to the downward offset condition. For the forward-propagating long wave, its amplitude oscillates by approximately $0.2 \mathrm{~m}$, suggesting the insensitive nature of 
the long wave to the upward offset shear current. The small amplitudes of the oscillating tail and amplitude-modulated wave packet in larger $\Delta$ cases indicate that the modulation could be weaker when $\Delta$ increased, and the weakening of the oscillating tail makes the amplitude-modulated wave packet more visible.

\subsection{The evolution of mode-2 ISWs in opposing and polarity-reversal background shear currents}

The modulation of mode-2 ISWs in the following (control experiment) and opposing shear currents was compared. In case D1, the background shear current oriented against the mode-2 ISW. The general pattern of the forward-propagating long wave, oscillating tail and amplitude-modulated wave packet were similar to those in the control experiment. In this opposing case D1, the amplitudes of the forwardpropagating long wave and oscillating tail were not significantly affected. For the mode-2 ISW, its amplitude also remains nearly unchanged between the following and opposing cases, while the amplitude-modulated wave packet's amplitude decreased from $1.85 \mathrm{~m}$ (following case) to $1.09 \mathrm{~m}$ in the opposing case (figure not shown). These results suggest that only the amplitude-modulated wave packet is sensitive to the orientation of the background shear current. The modulation of mode-2 ISWs in the polarity-reversal shear current was also compared to the control experiment. In case $\mathrm{P} 1$, the polarity-reversal background shear current was initialized in the model. The properties of the wave structures in case P1 and the control experiment were compared and no significant differences were found. Only the polarity of the forward-propagating long wave, oscillating tail and amplitude-modulated wave packet are reversed in case P1 (figure not shown). This result indicates that the polarity of those shear-induced wave structures is closely related to the polarity of the background shear current.

\subsection{The evolution of mode-2 ISWs in background shear currents with varied magnitudes}

The modulations of mode- 2 ISWs in variable magnitudes of shear currents were characterized. The magnitude of the background shear current was varied from $0.5 U_{\mathrm{d}}$ to 2.5 $U_{\mathrm{d}}$ from case $\mathrm{U} 1$ to case $\mathrm{U} 5$ to study its influence on the evolution of the mode-2 ISW. In case U5 (Fig. 9), a relatively larger amplitude forward-propagating long wave was observed at $20 T$ (Fig. 9c). The mode-2 ISW became inconspicuous at $50 T$ (Fig. 9d), while an amplitude-modulated wave packet propagated clearly. The increasing magnitude of the background shear current leads to smaller amplitudes of the mode-2 ISW in both the upper and lower parts. In the larger-magnitude case, the amplitude-modulated wave packet and forward-propagating long wave were significantly strengthened, and their amplitudes reached 3.75 and $0.38 \mathrm{~m}$ (case U5), respectively. In contrast, a larger magnitude did
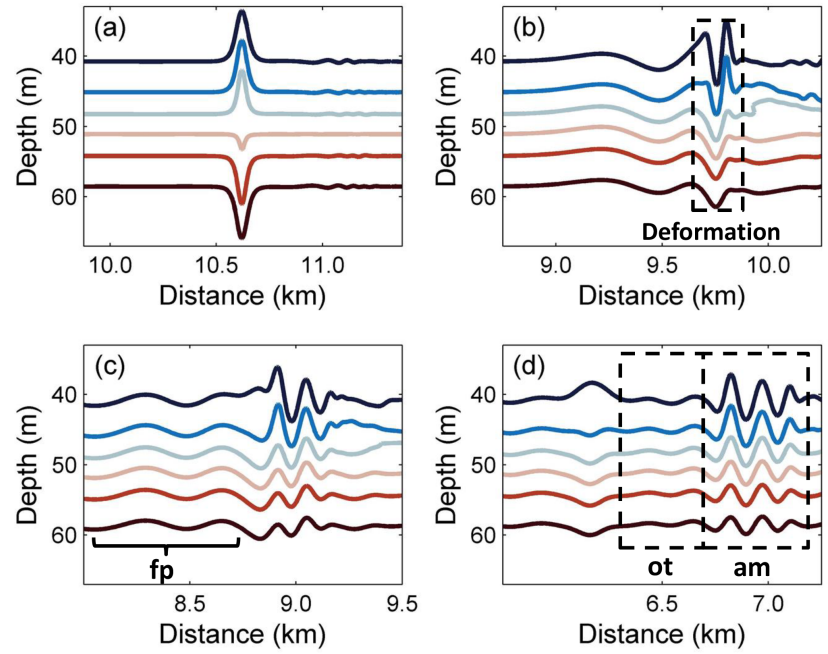

Figure 9. The evolution processes of the mode-2 ISW in case U5 for the different times (a) $0 \mathrm{~T}$, (b) $10 \mathrm{~T}$, (c) $20 \mathrm{~T}$, and (d) $50 \mathrm{~T}$, where "am", "fp" and "ot" denote the amplitude-modulated wave packet, forward-propagating long wave and oscillating tail, respectively.

not make the amplitude of the oscillating tail continue to increase. In the larger-magnitude case, the oscillating tail was unable to be clearly observed. Its amplitude becomes smaller $(0.25 \mathrm{~m})$ than that of the forward-propagating long wave $(0.38 \mathrm{~m})$. In summary, all three shear-induced waves are sensitive to the magnitude of the shear current.

\subsection{The evolution of mode-2 ISWs in background shear currents with varied thicknesses of shear}

The thickness of the shear layer $h_{\mathrm{s}}$ was also varied to investigate its effect on the modulation of mode-2 ISWs (cases $\mathrm{H} 1$ to H5). In comparison among these cases, the forwardpropagating long wave's amplitude decreased with larger $h_{\mathrm{s}}$, reaching $0.17 \mathrm{~m}$ in case $\mathrm{H} 5$ with $2.5 h_{\mathrm{s}}$. The amplitudemodulated wave packet and oscillating tail both decrease to 1.33 and $0.42 \mathrm{~m}$ in amplitude in larger $h_{\mathrm{s}}$ (case H5), respectively, while the mode-2 ISW's amplitude reaches $7.95 \mathrm{~m}$ (figure not shown). This result shows that the background shear current with smaller $h_{\mathrm{s}}$ could only moderately deform the mode-2 ISW. As a result, all three shear-induced wave structures are sensitive to the variation in the thickness of the shear layer.

\subsection{The relationship between the evolution process and variable parameters of background shear currents}

The amplitudes of the forward-propagating long wave, oscillating tail and amplitude-modulated wave packet in varied background shear currents are summarized to investigate their sensitivity to the varied background shear currents. The amplitudes of the forward-propagating long wave and amplitude-modulated wave packet are proportional to the 

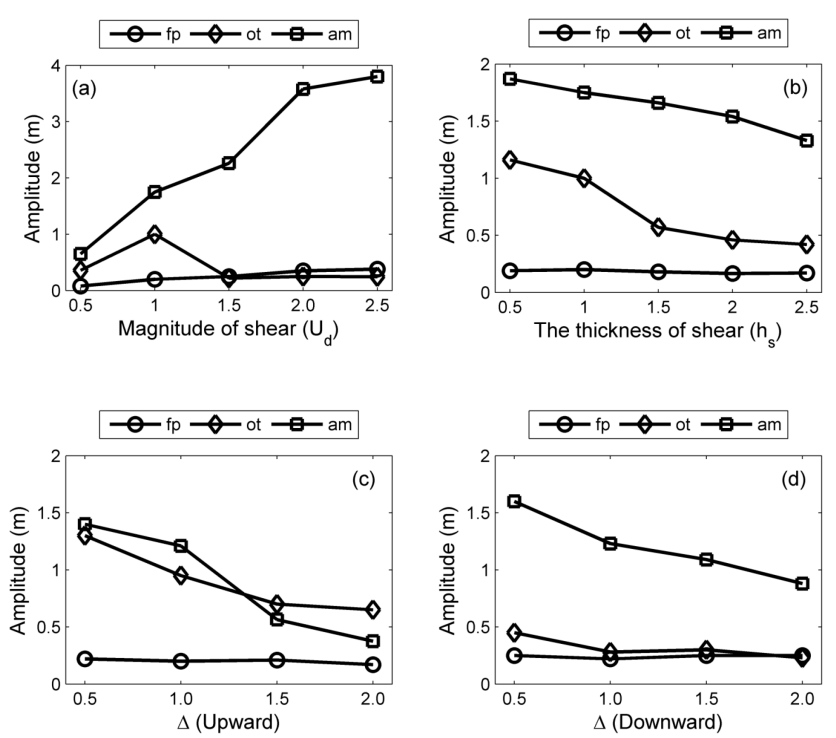

Figure 10. The summarized results of the amplitudes of the forward-propagating long wave (denoted by "fp"), oscillating tail (denoted by "ot") and amplitude-modulated wave packet (denoted by "am") with the presence of (a) varied magnitudes of shear currents at $40 T$, (b) varied thicknesses of shear currents at $40 T$, (c) upward offset background shear currents at $30 T$ and (d) downward offset background shear currents at $30 \mathrm{~T}$.

magnitude of the background shear current, but the oscillating tail is insensitive to the higher magnitude of the background shear current (Fig. 10a). The amplitudes of the oscillating tails and amplitude-modulated wave packet are inversely proportional to the thickness of the shear layer, and the forward-propagating long wave decreased slightly in amplitude with increasing $h_{\mathrm{s}}$ (Fig. 10b). To reveal the effects of the $\Delta$ on those shear-induced wave structures, a comparison of different cases from $\Delta=0$ (case O5) to $\Delta=2$ (case O9) was given (Fig. 10d). The modulation caused by background shear currents was weakened as the $\Delta$ increased, corresponding to the decreased amplitude of the amplitude-modulated wave packet and oscillating tail. The amplitude-modulated wave packet has the highest amplitude among all cases compared to those of the other two wave forms. The amplitude of the amplitude-modulated wave packet decreased from 1.8 to $1 \mathrm{~m}$ monotonically, and the amplitudes of the oscillating tails decreased from 0.85 to $0.25 \mathrm{~m}$ between case O5 $(\Delta=0)$ and case $\mathrm{O} 7(\Delta=1)$ but remained stable between case $\mathrm{O} 7$ $(\Delta=1)$ and case $\mathrm{O} 9(\Delta=2)$, indicating that the amplitudemodulated wave packets were more sensitive to the $\Delta$ than the oscillating tail. As expected, the ratio between the amplitude of the modulated wave packet and oscillating tails increased from 2.1 in case $\mathrm{O} 5$ to 4 in case $\mathrm{O} 9$, so the amplitudemodulated wave packet became more distinct in case O9. In contrast, the forward-propagating long wave was barely affected by $\Delta$ and remained constant at approximately $0.2 \mathrm{~m}$ in all cases. A similar variation trend could be found in the upward offset cases (Fig. 10c). The amplitudes of the oscillating tail and amplitude-modulated wave packet decreased monotonically as the shear current was offset upward. The forward-propagating long wave was barely affected by $\Delta$ and remained constant at approximately $0.2 \mathrm{~m}$ in all offset cases. This divergence of sensitivity between the forwardpropagating long wave, amplitude-modulated wave packet and oscillating tail could be related to their generation mechanisms, which are discussed in Sect. 5.

\section{Energy analyses}

\subsection{Calculation of energy}

The evolution of the mode-2 ISWs in the presence of shear currents was analysed quantitatively in terms of energy. The available potential energy (APE) and kinetic energy (KE) for a region were calculated based on the method suggested by Lamb (2010):

$$
\begin{aligned}
& \mathrm{KE}=\int_{x_{1}-\mathrm{H}}^{x_{\mathrm{r}}} \int_{x_{1}}^{0} \rho_{0}\left(u^{2}+w^{2}\right) \mathrm{d} x \mathrm{~d} z, \\
& \mathrm{APE}=\int_{x_{1}-\mathrm{H}}^{0} \int^{x_{\mathrm{r}}}(\rho-\bar{\rho}) g z \mathrm{~d} x \mathrm{~d} z,
\end{aligned}
$$

and the total energy $E$ is written as

$E=\mathrm{KE}+\mathrm{APE}$,

where $\bar{\rho}$ is the reference density extracted from the initial field, $\rho_{0}$ is the averaged density and $\rho$ is the fluid density. $x_{\mathrm{r}}$ and $x_{1}$ are the boundary locations of the integration region, and the $x$ satisfies $x_{1} \leq x \leq x_{\mathrm{r}}$. During the calculation of the wave energy, $x_{\mathrm{r}}$ and $x_{1}$ denote the left and right boundaries, respectively, where the available potential energy flux equals zero (Lamb, 2010). $u$ and $w$ are the horizontal and vertical velocities induced by the wave, respectively. The total energy of the initial mode-2 ISW just before the introduction of the shear calculated by the above expressions was $146.2 \mathrm{KJ} \mathrm{m}^{-1}$.

Using the method introduced by Lamb and Nguyen (2009), we set two transects at the front and rear edges of the mode-2 ISW to compute the energy fluxes radiating from the ISW. The total energy flux through a transect is

$E_{\mathrm{f}}=\mathrm{KE}_{\mathrm{f}}+\mathrm{APE}_{\mathrm{f}}+W$,

where $\mathrm{KE}_{\mathrm{f}}, \mathrm{APE}_{\mathrm{f}}$ and $W$ are the kinetic, available potential and pressure perturbation energy fluxes, respectively. They are written as 

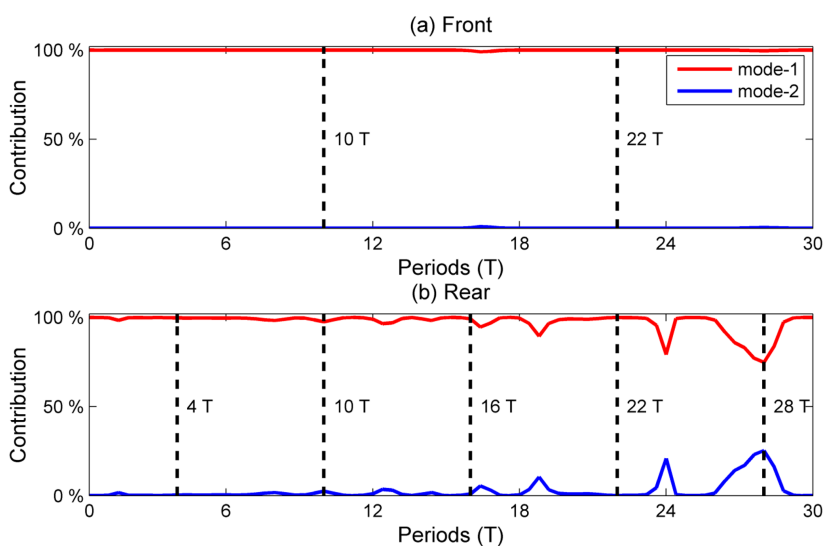

Figure 11. Percent contributions of mode- 1 and mode- 2 to the total kinetic energy in the control experiment (case O5 with $\Delta=0$ ) from 0 to $30 T$ at the (a) front and (b) rear of the mode-2 ISW; the dash lines indicate the cross sections where the modal structures of waves were shown in Fig. 12.

$\mathrm{KE}_{\mathrm{f}}=\int_{-\mathrm{H}}^{0} u E_{k e} \mathrm{~d} z$,

$\mathrm{APE}_{\mathrm{f}}=\int_{-\mathrm{H}}^{0} u E_{\text {ape }} \mathrm{d} z$,

$W=\int_{-\mathrm{H}}^{0} u p_{\mathrm{d}} \mathrm{d} z$,

where $u$ is the horizontal velocity induced by mode-2 ISWs, $E_{\mathrm{ke}}$ and $E_{\mathrm{ape}}$ are kinetic energy and available potential energy, and $p_{\mathrm{d}}$ is the pressure perturbation relative to the reference state (Lamb and Nguyen, 2009).

\subsection{The cascading process of energy}

To understand better how the mode-2 ISW is modulated with the presence of shear current and to determine the nature of the whole wave system, the EOF (empirical orthogonal function) method was applied to the modal decomposition. It is commonly used for mode decomposition and space-timedistributed dataset examination in oceanography (Venayagamoorthy and Fringer, 2007), especially with strong nonlinearity properties, where the traditional normal mode decomposition is not suitable (Venayagamoorthy and Fringer, 2007). Because of the shear effect on the mode-2 ISWs, the energy can cascade into all the modes, including mode-1 and higher modes. The vertical and horizontal kinetic energy modal distributions of different wave forms shed from the mode-2 ISW in case O5 are shown in Fig. 11 and the modal structures on waves (Talipova et al., 2011) for different times

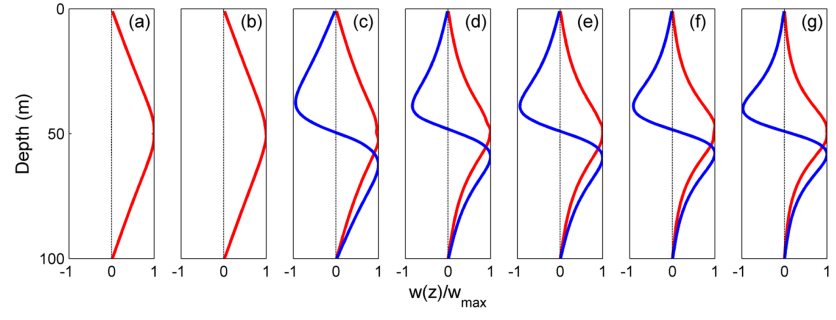

Figure 12. The modal structures on mode-1 (red solid line) and mode-2 (blue solid line) waves in front of the mode-2 ISW at (a) $10 T$ and (b) $22 T$ and in the rear of the mode-2 ISW at (c) $4 T$, (d) $10 T$, (e) $16 T$, (f) $22 T$ and (g) $28 T$ for the control experiment (case O5).

are given in Fig. 12. In the forward-propagating long waves, the kinetic energy was all in the mode- 1 form because of its propagation in front of the ISWs, indicating the generation of the forward-propagating long waves corresponds to a cascading process from higher to lower modes. Figure 11 shows that the energy was mainly mode- 1 in the oscillating tail and the amplitude-modulated wave packet, but weak mode- 2 signals were also present. The presence of mode- 2 energy for the oscillating tail is reasonable because they have shorter wavelengths and slower phase speeds than the mode2 ISW and propagate following the mode-2 ISW (Akylas and Grimshaw, 1992; Vlasenko et al., 2010). The modal structures of forward-propagating long waves for different times show its mode- 1 nature was stable during the evolution of mode-2 ISWs in the background shear current (Fig. 12a and b). In the rear of the mode-2 ISW, the modal structures of trailing waves transformed slightly with time (Fig. 12c-g), and the wave-induced currents are more and more concentrated around the pycnocline when the amplitude-modulated wave packet propagates far away from the mode-2 ISW.

\subsection{Energy loss of mode-2 ISWs}

The energy loss of mode-2 ISWs in the control experiment was investigated in detail to demonstrate the corresponding energy changing process during the modulation and compared with the observations of Shroyer et al. (2010). In this case, $\sim 36 \%$ of the total energy of the mode- 2 ISW was lost by $30 T$, corresponding to a propagation of $1.86 \mathrm{~km}$; part of that energy was transferred to shear-induced wave forms. During the first $30 T$, the average energy loss rate was $9 \mathrm{~W} \mathrm{~m}^{-1}$. Modulated by the background shear current, the mode-2 ISW exhibits a highly dissipated nature, and the high energy loss rate is comparable to that of the longer mode-1 ISW (Lamb and Farmer, 2011; Shroyer et al., 2010). This quantitative result was consistent with the observation data (Shroyer et al., 2010).

We further calculated the radiating energy flux (Fig. 13) to investigate the detailed energy transport. The pressure perturbation generally makes the largest instantaneous contribu- 

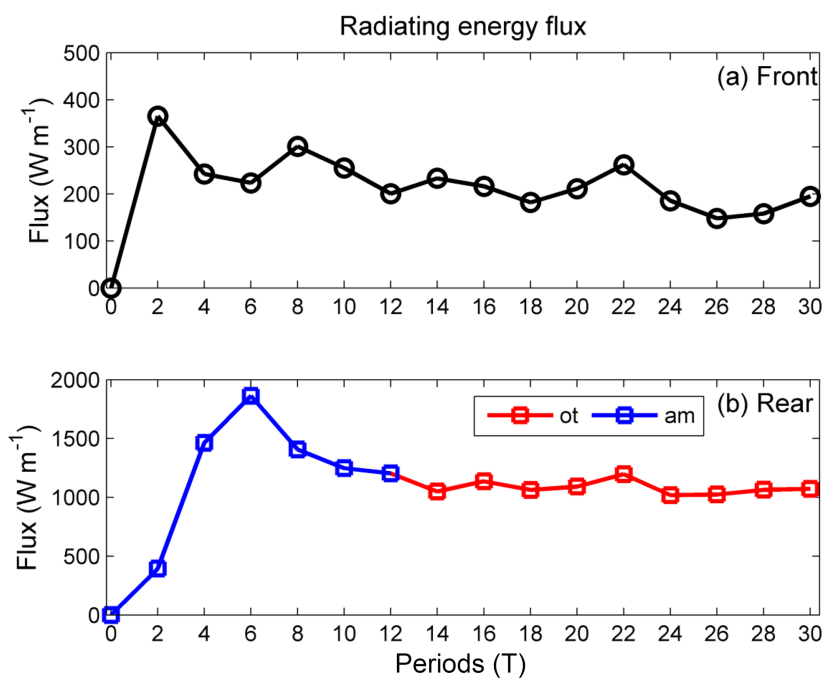

Figure 13. Vertical integrals of the radiating energy flux in the (a) front and (b) rear transects of the mode-2 ISW in the control experiment (case O5 with $\Delta=0$ ) at different times, where "ot" denotes the oscillating tail and "am" denotes the amplitude-modulated wave packet.

tions to the total energy flux (Lamb and Nguyen, 2009; Venayagamoorthy and Fringer, 2007). For an ISW, the pressure perturbation term could be dominant (Lamb, 2007). Since we focused on the energy loss of the mode-2 ISW, only a total energy flux was analysed in the following paragraph. The radiating energy flux in the front transect slowly decreased, indicating the forward-propagating long wave drains the energy of the mode-2 ISW at a decreasing rate in the presence of a background shear current. In the rear transect, the radiating energy flux decreased from 1.8 to $1.2 \mathrm{KW} \mathrm{m}^{-1}$ before stabilizing at approximately $1.0 \mathrm{KW} \mathrm{m}^{-1}$ above $12 \mathrm{~T}$. The energy flux at the crest of the amplitude-modulated wave packet and the oscillating tail ranged from 1.0 to $2.0 \mathrm{KW} \mathrm{m}^{-1}$ (Blue solid line in Fig. 13) and $1.0 \mathrm{KW} \mathrm{m}^{-1}$ to $1.1 \mathrm{KW} \mathrm{m}^{-1}$, respectively. Combining with the evolution process, the high radiating flux before $12 T$ indicates the generation process of the amplitude-modulated wave packet and that the relatively low radiating energy flux above $12 T$ is caused by the generation of an oscillating tail.

The total energy of the mode-2 ISWs at different times in the control experiment is shown in Fig. 14. From 0 to $6 T$, the averaged energy loss rate was $18.4 \mathrm{~W} \mathrm{~m}^{-1}$. This period corresponded to the generation of the amplitude-modulated wave packet and forward-propagating waves, during which a deformation was caused by shear to the aft of the ISW. The averaged energy loss rates from 6 to $12 T$ decreased. This stage contained the exit of the amplitude-modulated wave packet, which occurred at approximately $10 T$ (Fig. 4 and Fig. 7), and the generation of the oscillating tail. In this case, $\sim 34 \%$ of the total energy $\left(52.34 \mathrm{KJ} \mathrm{m}^{-1}\right)$ was lost at an average rate of $14.8 \mathrm{~W} \mathrm{~m}^{-1}$ from 6 to $12 T$, and some of the

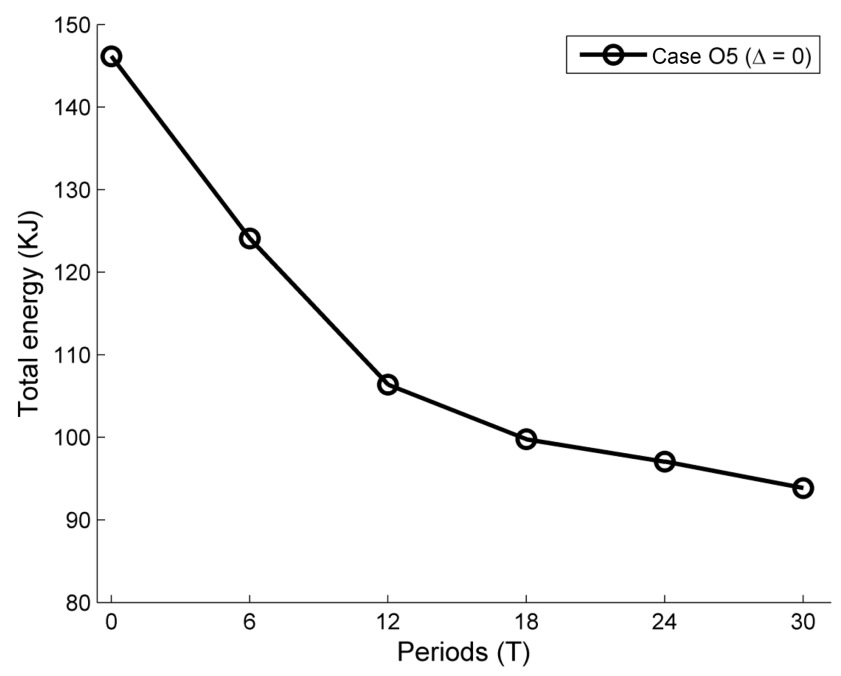

Figure 14. The total energy of the ISW at the different times in case O5.

energy transferred to the amplitude-modulated wave packet. Combining the results of the energy flux in the front and rear of the mode-2 ISW (Fig. 13), i.e. in the early stage of modulation, the amplitude-modulated wave packet could make a larger contribution to the energy transfer process. After the amplitude-modulated wave packet shed from the mode2 ISWs, sharply decreased loss rates could be seen in 12 $18 T$, during which the energy loss was caused by forwardpropagating long waves and the oscillating tail. The shear currents continuously sustained the development of the oscillating tail and the forward-propagating long waves. Thus, these two forms could slowly drain the energy of the ISW, with an average rate of $3.5 \mathrm{~W} \mathrm{~m}^{-1}$. In the following periods, with the forward-propagating long waves and oscillating tails, the ISWs decayed with a relatively low rate. This was reinforced by a similar result given by Olsthroon et al. (2013).

\subsection{The relationship between the energy loss of mode-2 ISWs and variable parameters of background shear currents}

We summarized the effect of variable parameters of shear currents on the energy loss of mode-2 ISWs during the modulation (Fig. 15). The polarity and the direction of the background shear current have a minor effect on the energy loss of mode-2 ISWs (figure not shown). The energy loss of the mode-2 ISW was proportional to the magnitude of the shear current, but inversely proportional to the thickness of the shear layer (Fig. 15a and b). For case U5, $78.04 \mathrm{KJ} \mathrm{m}^{-1}$ energy loss in $30 T, \sim 53 \%$ of the total energy of mode- 2 ISWs, and the averaged energy loss rate was $13 \mathrm{~W} \mathrm{~m}^{-1}$, indicating the magnitude of shear could significantly increase the energy loss of mode- 2 ISWs. In contrast, for case H5, $42.05 \mathrm{KJ} \mathrm{m}^{-1}$ energy loss in $30 T, \sim 29 \%$ of the total en- 

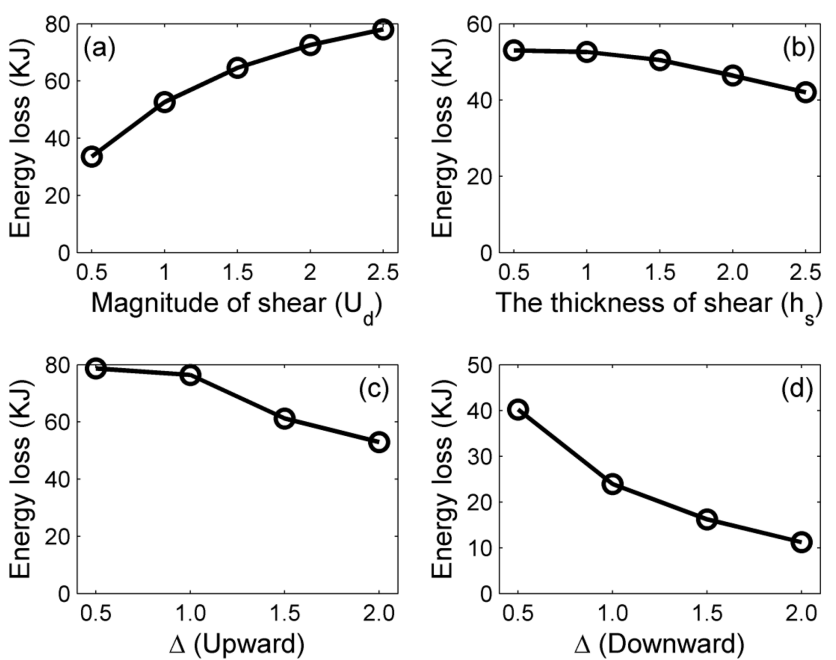

Figure 15. The summarized results of the energy loss of the mode2 ISW at $30 T$ with the presence of (a) varied magnitudes of shear currents, (b) varied thicknesses of shear currents, (c) upward offset background shear currents and (d) downward offset background shear currents.

ergy of mode-2 ISWs, and the averaged energy loss rate was $7 \mathrm{~W} \mathrm{~m}^{-1}$, showing that a larger thickness of shear has the opposite effect on the energy loss of mode- 2 ISWs. In the offset background shear currents, the energy loss of mode-2 ISWs monotonically decreased with an increasing $\Delta$, showing a symmetric trend in both upward and downward offset cases (Fig. 15c and d). Therefore, the energy losses of mode-2 ISWs are sensitive to the magnitude, thickness and offset extent, but insensitive to the polarity and direction of the background shear current. We further found that the energy losses of mode-2 ISWs in upward conditions were larger compared to the downward conditions. This phenomenon is caused by the asymmetry of wave-induced shear. When the shear layer moves up or down, the background current could weaken the shear in the upper layer or strengthen the shear in the lower layer, causing the difference in energy losses of mode-2 ISWs.

\section{Discussions}

We compared our results of the control experiment (case O5) with the observation of Shroyer et al. (2010) for validation. The wavelengths and amplitudes of the initialized mode-2 ISWs were selected to be comparable to the observation of Shroyer et al. (2010) on the New Jersey Shelf. A depression wave at the rear of the ISW in the first transect of wave Jasmine was similar to the wave form around $10 T$ in the numerical simulation (Fig. 3a in Shroyer et al., 2010). Thus the first, second and third transects of wave Jasmine corresponded to 10,23 and $38 T$ in case O5, respectively. The energy loss rates in 10 and $23 T$ were 14.8 and $2.3 \mathrm{~W} \mathrm{~m}^{-1}$, and the averaged energy loss rate between 10 and $38 T$ was $4.1 \mathrm{~W} \mathrm{~m}^{-1}$; they were in the same scale as the corresponding observations. Between 10 and $38 T$, $\sim 16 \%$ of mode- 2 ISW total energy lost, it was a little smaller than the typical observation results. A relatively high energy loss rate and a large-amplitude oscillating tail in the third transect of the field observations could probably be attributed to the effect of a shoaling pycnocline (Shroyer et al., 2010) since the enhancement of the asymmetries in stratification could increase the energy loss of the wave during the propagation of mode-2 ISWs (Carr et al., 2015; Olsthoorn et al., 2013).

We also compared our results with Stastna et al. (2015), who investigated the mode-2 ISW interaction with mode-1 ISWs at the same scale. The authors concluded that the shear current is vital, while the deformation of the pycnocline only slightly altered the structure of the mode-2 ISW. For our results, we focused on the effect of shear currents induced by baroclinic eddies, baroclinic tides or wind. We found a deformation of the mode-2 ISW and it illustrated asymmetry during the modulation in the presence of background shear currents; it is coincident with the conclusion given by Stastna et al. (2015).

Then, we further discussed the characteristics of shearinduced waves. In our simulations, the modulation of mode-2 ISWs in the presence of shear currents excites the amplitudemodulated wave packet with characteristics of breather-like internal waves (Terletska et al., 2016). Internal breather waves are periodically pulsating, isolated wave forms; they are also a type of steady-state wave solution of the extended Korteweg-de Vries equation (Lamb et al., 2007), and have been found to exist in the real ocean (Vlasenko and Stashchuk, 2015). We introduced the definition of a breather by Clarke et al. (2000) to clarify the characteristics of amplitude-modulated wave packets. The envelope lines of the amplitude-modulated wave packet in case $\mathrm{O5}$ are shown in Fig. 16. Inside the envelope lines, the oscillatory pulses freely oscillate, satisfying the breather definition. Additionally, the energy inside the envelope was calculated and remains nearly constant from 12 to $28 \mathrm{~T}$. Similar to case $\mathrm{O5}$, the characteristics and energy loss of the amplitudemodulated wave packet for case 09 were also similar with the breather definition. Similar results were revealed by Terletska et al. (2016); the interaction of mode-2 internal waves with a step-like topography could induce the generation of BLIWs (breather-like internal waves), providing a possibility of the breather generation in a thin intermediate layer with a range of intermediate wavelength.

An oscillating tail induced by shear was also observed in similar studies. The generation of this feature could be related to the shear, and the tail was sustained by continuous energy input. The presence of the background shear current modulated the mode- 2 ISW and induced the continuous energy transfer process from the main wave to the oscillating tail, supporting its existence. Forward-propagating long waves were also observed by Yuan et al. (2018), who 


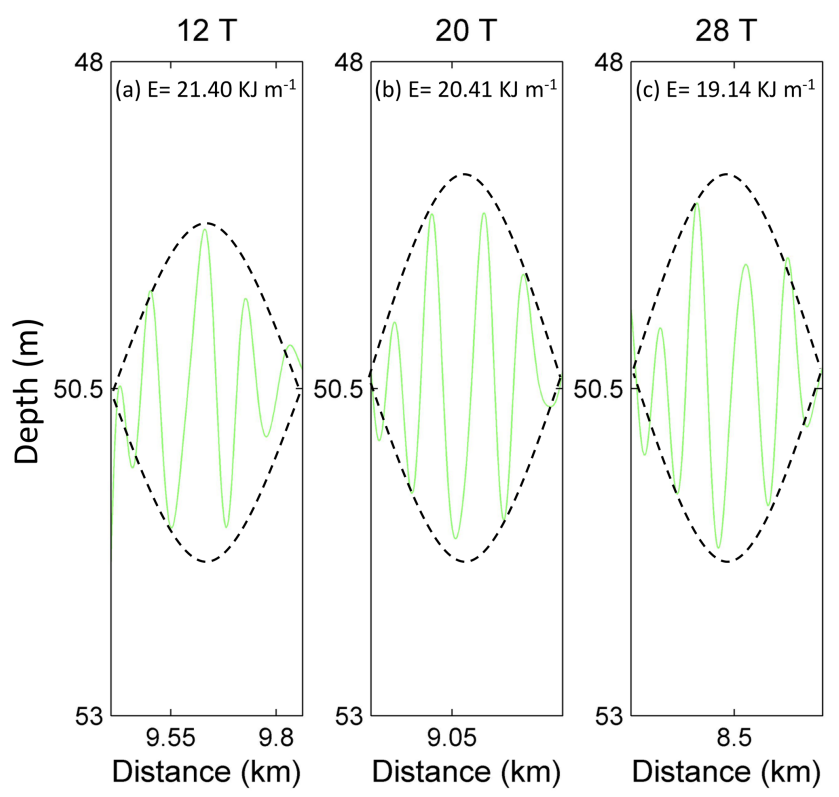

Figure 16. The envelopes of the amplitude-modulated wave packet in case $\mathrm{O} 5(\Delta=0)$ at (a) $12 \mathrm{~T}$, (b) $20 \mathrm{~T}$ and (c) $28 \mathrm{~T}$, where the mean density isopycnals of the upper and lower layers (green line) are plotted.

found that some small but significant long wavelength mode1 waves appeared ahead of mode-2 ISWs. The forwardpropagating long wave was generated by the collapse of mixing induced by shear instability, and it could drain the energy of mode-2 ISWs at a decreasing rate, leading to an inevitable energy loss of those mode-2 ISWs in the presence of background shear. The results in Sect. 3.7 show that the amplitude of the forward-propagating long wave is proportional to the magnitude of the shear current, indicating that the forward-propagating long wave was affected by the strength of the shear. $\Delta$ denotes the offset extent of the background shear current, and the strength of the shear remains unchanged when $\Delta$ varied. Therefore, the forwardpropagating long wave was insensitive to variation in $\Delta$.

The mechanism of adjustment during the modulation has been reviewed. The superposition of an initially stable shear current and the mode-2 ISW induced a low $R i$ region with a minimum value of less than 0.01 in our simulation, indicating a possible development of shear instability (Barad and Fringer, 2010). The ISW tends to adjust gradually and adapt to the new background conditions. The vorticity and $R i$ of the adjustment process for the mode-2 ISW in case O5 are shown in Fig. 17. The $R i$ values are larger than 0.25 before $0.8 T$. After $0.8 T$, due to the shear currents and weakened stratification, the lowest $R i$ values decrease below 0.01 and are accompanied by increased vorticity around the low $R i$ region, indicating the generation of shear instability (Pawlak and Armi, 1998). The overturning in isopycnals could also be observed in the corresponding low Ri region (Fig. 18). Then,
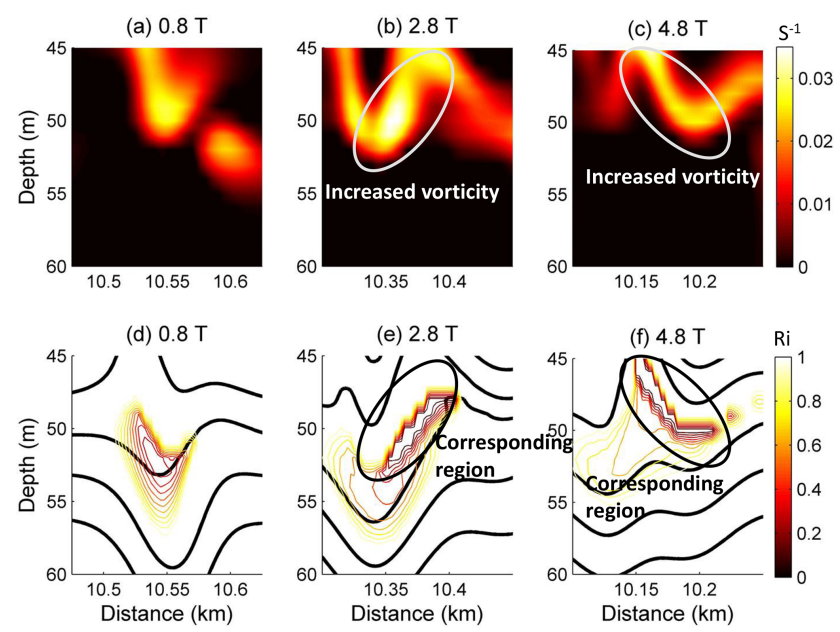

Figure 17. The vorticity spatial distributions for case O5 $(\Delta=0)$ at (a) $0.8 T$, (b) $2.8 T$, (c) and $4.8 T$, and the corresponding $R i$ values (ranges from 0 to 1 ) at (d) $0.8 T$, (e) $2.8 T$, and (f) $4.8 T$.

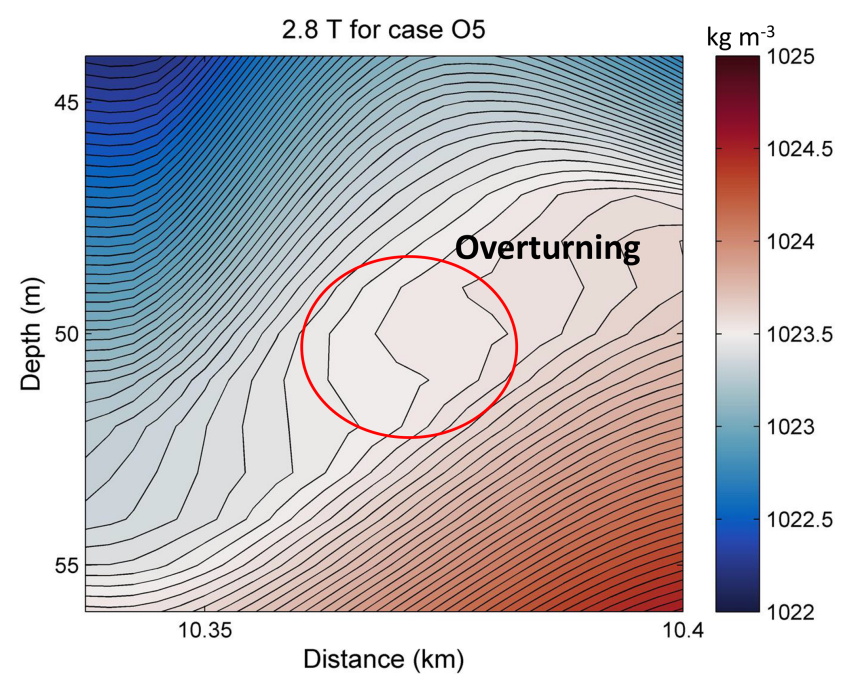

Figure 18. The density contour plot at $2.8 T$ for case O5.

the $R i$ values increased to larger than 0.25 , and the stratification is restored above $6.8 T$ (figure not shown). For case O9 (Fig. 19), before $0.8 T$, the $R i$ values are also larger than 0.25 . They decrease below 0.01 near the depths of the shear current after $0.8 T$, which is accompanied by increased vorticity and weakened stratification, indicating the occurrence of the shear instability. The stratification is restored and the $R i$ values increased to larger than 0.25 after $2 T$ (figure not shown). Compared to case O5, the region with low $R i$ and increased vorticity was smaller in case $\mathrm{O} 9$, making the instability process less apparent, and the shear instability for case $\mathrm{O} 5$ occurs at the same time but lasts longer than that for case 09. Those comparisons illustrated that the adjustment of mode-2 ISWs modulated by the shear current are more energetic in overlap cases compared to offset cases. 

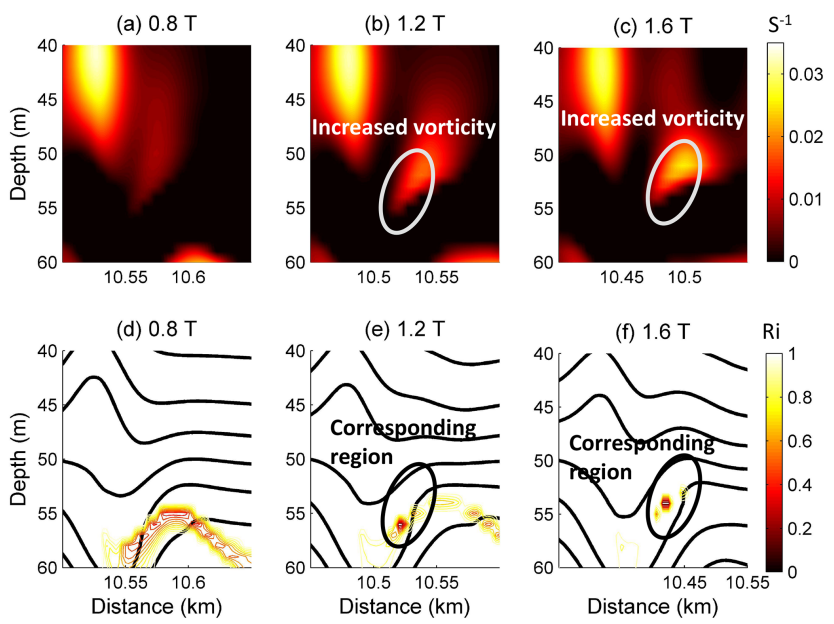

Figure 19. The vorticity spatial distributions for case $\mathrm{O} 9(\Delta=2)$ at (a) $0.8 T$, (b) $1.2 T$, and (c) $1.6 T$, and the corresponding $R i$ values (ranges from 0 to 1 ) at (d) $0.8 T$, (e) $1.2 T$, and (f) $1.6 T$.

As for the long-term behaviour, the mode-2 ISW could adjust itself to adapt to new background conditions and experience a dramatic transformation with disintegration into a wave train (Grimshaw et al., 2010; Yuan et al., 2018). In our simulation, the mode-2 ISW was observed to adjust itself to the new background condition with a shear current. The high energy loss rate is in agreement with the observation by Shroyer et al. (2010). However, the mode-2 ISW might not be able to survive for a long time in situ because the background conditions in the real ocean could be more complex and vary with time, leading to a background condition where a stable solution of mode-2 ISWs does not exist.

\section{Conclusions}

We have presented the evolution process of mode-2 ISWs modulated by varied background shear currents with the MITgcm in this study. It was illustrated that the adjustment of the mode-2 ISWs in the presence of background shear currents occurs through the generation of forward-propagating long waves, an amplitude-modulated wave packet, and an oscillating tail.

For comparison with the observation, a control experiment was conducted (case O5); $\sim 36 \%$ of the total energy of the mode-2 ISW was lost at an average rate of $9 \mathrm{~W} \mathrm{~m}^{-1}$, and this rate was in agreement with the observation of Shroyer et al. (2010). The mode-2 ISWs are highly dissipated in the presence of shear currents, and it was consistent with the hypothesis given by Shroyer et al. (2010). In addition, five sets of experiments were introduced to assess the sensitivity of the evolution process to different properties of the background shear currents in order to get a general conclusion. We found that the polarity and direction of the background shear current have a minor effect on the evolution of the mode-2 ISW. The amplitudes of the forward-propagating long wave and amplitude-modulated wave packet as well as the decaying of mode-2 ISWs' energy are proportional to the magnitude of the shear but inversely proportional to the thickness of the shear layer. We also found that the oscillating tail and amplitude-modulated wave packet show a symmetric variation trend in both offset upward and downward conditions, while the forward-propagating long wave was insensitive to the offset extent of background shear current, and the shear layers centered at the mid-depth of pycnocline had a much more pronounced energy loss of the mode-2 ISW compared to those cases where the shear layer centered away from the mid-depth of the pycnocline.

In future work, a possible avenue is the evolution of a mode-2 ISW in the time-varied background shear current, and the wave-mean flow interaction in this complicated flow field, revealing the energy exchange process between waves and mean flow. The other possible direction is the investigation of a combination effect which is closer to field observations on the evolution of the mode- 2 ISW, including the effect of background shear current, varying topography and shoaling pycnocline.

Code and data availability. The MITgcm program can be downloaded from the websites at http://mitgcm.org (last access: 20 June 2018). The EOF codes can be downloaded from http:// cn.mathworks.com/matlabcentral/fileexchange/17915-pcatool (last access: 20 June 2018). The simulation data deposit for this paper needs a high-capacity disk and is available on request to Zhenhua Xu by email.

Competing interests. The authors declare that they have no conflict of interest.

Special issue statement. This article is part of the special issue "Extreme internal wave events". It is a result of the EGU, Vienna, Austria, 23-28 April 2017.

Acknowledgements. Funding for this study was provided by the National Key Research and Development Program of China (nos. 2016YFC1401404 and 2017YFA0604102), the Scientific and Technological Innovation Project financially supported by QNLM (no. 2016ASKJ12), the National Natural Science Foundation of China (41528601, 41676006, 41421005, 41576189), the Youth Innovation Promotion Association, CAS, CAS Interdisciplinary Innovation Team "Oceanic Mesoscale Processes and Ecology Effects", the Key Research Program of Frontier Science, CAS (QYZDB-SSW-DQC024), and the Strategic Pioneering Research Program of CAS (XDA11020104, XDA11020101). This study was supported by the High Performance Computing Center at the IOCAS. Constructive and helpful comments from the editor, Tatiana Talipova, Magda Carr, and one anonymous referee are gratefully acknowledged. 
Edited by: Marek Stastna

Reviewed by: Tatiana Talipova, Magda Carr, and one anonymous referee

\section{References}

Akylas, T. R. and Grimshaw, R. H. J.: Solitary internal waves with oscillatory tails, J. Fluid Mech., 242, 279-298, 1992.

Barad, M. F. and Fringer, O. B.: Simulations of shear instabilities in interfacial gravity waves, J. Fluid Mech., 644, 61-95, 2010.

Brandt, A. and Shipley, K. R.: Laboratory experiments on mass transport by large amplitude mode-2 internal solitary waves, Phys. Fluids, 26, 016602-82, https://doi.org/10.1063/1.4869101, 2014.

Carpenter, J. R., Balmforth, N. J., and Lawrence, G. A.: Identifying unstable modes in stratified shear layers, Phys. Fluids, 22, 054104, https://doi.org/10.1063/1.3379845, 2010.

Carr, M., Davies, P. A., and Hoebers, R. P.: Experiments on the structure and stability of mode-2 internal solitary-like waves propagating on an offset pycnocline, Phys. Fluids, 27, 046602, https://doi.org/10.1063/1.4916881, 2015.

Chen, G. Y., Liu, C. T., Wang, Y. H., and Hsu, M. K.: Interaction and generation of long - internal solitary waves in the South China Sea, J. Geophys. Res., 116, 1-7, https://doi.org/10.1029/2010JC006392, 2011.

Clarke, S., Grimshaw, R., Miller, P., Pelinovsky, E., and Talipova, T.: On the generation of solitons and breathers in the modified Korteweg-de Vries equation, Chaos: An Interdisciplinary, J. Nonlin. Sci., 10, 383-392, 2000.

Deepwell, D. and Stastna, M.: Mass transport by mode-2 internal solitary-like waves, Phys. Fluids, 28, 395-425, 2016.

Duda, T. F., Lynch, J. F., Irish, J. D., Beardsley, R. C., Ramp, S. R., Ching-Sang, C., Tswen Yung, T., and Yang, Y.-J.: Internal tide and nonlinear internal wave behavior at the continental slope in the northern South China Sea, IEEE J. Ocean. Eng., 29, 11051130, 2004.

Farmer, D., Li, Q., and Park, J. H.: Internal wave observations in the South China Sea: The role of rotation and non - Atmos.-Ocean, 47, 267-280, 2009.

Grimshaw, R., Pelinovsky, E., and Talipova, T.: Modelling internal solitary waves in the coastal ocean, Surv. Geophys., 28, 273-298, 2007.

Grimshaw, R., Pelinovsky, E., Talipova, T., and Kurkina, O.: Internal solitary waves: propagation, deformation and disintegration, Nonlin. Processes Geophys., 17, 633-649, https://doi.org/10.5194/npg-17-633-2010, 2010.

Helfrich, K. R. and Melville, W. K. On long nonlinear internal waves over slope-shelf topography, J. Fluid Mech., 167, 285308, 1986.

Helfrich, K. R. and Melville, W. K.: Long nonlinear internal waves, Annu. Rev. Fluid Mech., 38, 395-425, 2006.

Hüttemann, H. and Hutter, K.: Baroclinic solitary water waves in a two-layer fluid system with diffusive interface, Exp. Fluids, 30, 317-326, 2001.

Jackson, C. R., da Silva, J. C. B., Jeans, G., Alpers, W., and Caruso, M. J.: Nonlinear internal waves in synthetic aperture radar imagery, Oceanography, 26, 68-79, 2013.
Klymak, J. M. and Moum, J. N.: Internal solitary waves of elevation advancing on a shoaling shelf, Geophys. Res. Lett., 30, 315-331, https://doi.org/10.1029/2003GL017706, 2003.

Lamb, K. G.: Energetics of internal solitary waves in a background sheared current, Nonlin. Processes Geophys., 17, 553568, https://doi.org/10.5194/npg-17-553-2010, 2010.

Lamb, K. G.: Internal wave breaking and dissipation mechanisms on the continental slope/shelf, Ann. Rev. Fluid Mech., 46, 231254, 2014.

Lamb, K. G. and Farmer, D.: Instabilities in an internal solitary-like wave on the oregon shelf, J. Phys. Oceanogr., 41, 67-87, 2011.

Lamb, K. G. and Nguyen, V. T.: Calculating energy flux in internal solitary waves with an application to reflectance, J. Phys. Oceanogr., 39, 559-580, 2009.

Lamb, K. G., Polukhina, O. E., Talipova, T., Pelinovsky, E., Xiao, W., and Kurkin, A.: Breather generation in fully nonlinear models of a stratified fluid, Phys. Rev. E, 75, 046306, https://doi.org/10.1103/PhysRevE.75.046306, 2007.

Legg, S. and Adcroft, A.: Internal wave breaking at concave and convex continental slopes, J. Phys. Oceanogr., 33, 2224-2246, 2003.

Legg, S. and Huijts, K. M.: Preliminary simulations of internal waves and mixing generated by finite amplitude tidal flow over isolated topography, Deep Sea Res. II, 53, 140-156, 2006.

Legg, S. and Klymak, J.: Internal hydraulic jumps and overturning generated by tidal flow over a tall steep ridge, J. Phys. Oceanogr., 38, 1949-1964, 2008.

Liu, A. K., Chang, Y. S., Hsu, M. K., and Liang, N. K.: Evolution of nonlinear internal waves in the East and South China Seas, J. Geophys. Res., 103, 7995-8008, 1998.

Liu, A. K., Ramp, S. R., Zhao, Y., and Tang, T. Y.: A case study of internal solitary wave propagation during ASIAEX 2001, IEEE J. Ocean. Eng., 29, 1144-1156, 2004.

Liu, A. K., Su, F. C., Hsu, M. K., Kuo, N. J., and Ho, C. R.: Generation and evolution of mode-two internal waves in the South China Sea, Cont. Shelf Res., 59, 18-27, 2013.

Marshall, J., Hill, C., Perelman, L. T., and Adcroft, A.: Hydrostatic, quasi-hydrostatic, and nonhydrostatic ocean modeling, J. Geophys. Res., 102, 5733-5752, 1997.

Moum, J. N., Klymak, J. M., Nash, J. D., Perlin, A., and Smyth, W. D.: Energy transport by nonlinear internal waves, J. Phys. Oceanogr., 37, 1968-1988, 2006.

Olsthoorn, J., Baglaenko, A., and Stastna, M.: Analysis of asymmetries in propagating mode- 2 waves, Nonlin. Processes Geophys., 20, 59-69, https://doi.org/10.5194/npg-20-59-2013, 2013.

Pawlak, G. and Armi, L.: Vortex dynamics in a spatially accelerating shear layer, J. Fluid Mech., 376, 1-35, 1998.

Ramp, S. R., Yang, Y. J., Reeder, D. B., and Bahr, F. L.: Observations of a mode-2 nonlinear internal wave on the northern heng-chun ridge south of taiwan, J. Geophys. Res., 117, 3043, https://doi.org/10.1029/2011JC007662, 2012.

Salloum, M., Knio, O. M., and Brandt, A.: Numerical simulation of mass transport in internal solitary waves, Phys. Fluids, 24, 21110, 2012.

Shroyer, E. L., Moum, J. N., and Nash, J. D.: Mode 2 waves on the continental shelf: ephemeral components of the nonlinear internal wavefield, J. Geophys. Res., 115, 419-428, 2010. 
Stastna, M. and Lamb, K. G.: Large fully nonlinear internal solitary waves: The effect of background current, Phys. Fluids, 14, 29872999, 2002.

Stastna, M. and Peltier, W. R.: On the resonant generation of large-amplitude internal solitary and solitary-like waves, J. Fluid Mech., 543, 267-292, 2005.

Stastna, M., Olsthoorn, J., Baglaenko, A., and Coutino, A.: Strong mode-mode interactions in internal solitary-like waves, Phys. Fluids, 27, 046604, https://doi.org/10.1063/1.4919115, 2015.

Talipova, T. G., Pelinovsky, E. N., and Kharif, C.: Modulation instability of long internal waves with moderate amplitudes in a stratified horizontally inhomogeneous ocean, JETP Lett., 94, 182186, 2011.

Terletska, K., Jung, K. T., Talipova, T., Maderich, V., Brovchenko, I., and Grimshaw, R.: Internal breather-like wave generation by the second mode solitary wave interaction with a step, Phys. Fluids, 28, L03601-276, https://doi.org/10.1063/1.4967203, 2016.

Van der Boon, C. M.: Numerical modelling of internal waves in the Browse Basin, MSc Thesis, TU Delft, Delft, the Netherlands, 2011.

Venayagamoorthy, S. K. and Fringer, O. B.: On the formation and propagation of nonlinear internal boluses across a shelf break, $\mathrm{J}$. Fluid Mech., 577, 137-159, 2007.

Vlasenko, V. and Stashchuk, N.: Internal tides near the Celtic Sea shelf break: A new look at a well known problem, Deep Sea Res. I, 103, 24-36, 2015.

Vlasenko, V., Stashchuk, N., Guo, C., and Chen, X.: Multimodal structure of baroclinic tides in the South China Sea, Nonlin. Processes Geophys., 17, 529-543, https://doi.org/10.5194/npg-17529-2010, 2010.
Wang, J., Ingram, R. G., and Mysak, L. A.: Variability of internal tides in the Laurentian Channel, J. Geophys. Res., 96, 1685916875, https://doi.org/10.1029/91JC01580, 1991.

$\mathrm{Xu}, \mathrm{Z}$. H., Yin, B., Hou, Y., and Xu, Y.: Variability of internal tides and near-inertial waves on the continental slope of the northwestern South China Sea, J. Geophys. Res.-Oceans, 118, 197-211, https://doi.org/10.1029/2012JC008212, 2013.

Xu, Z. H., Liu, K., Yin, B., Zhao, Z., Wang, Y., and Li, Q.: Longrange propagation and associated variability of internal tides in the South China Sea, J. Geophys. Res.-Oceans, 121, 8268-8286, https://doi.org/10.1002/2016JC012105, 2016.

Yang, Y. J., Fang, Y. C., Chang, M. H., Ramp, S. R., Kao, C. C., and Tang, T. Y.: Observations of second baroclinic mode internal solitary waves on the continental slope of the northern south china sea, J. Geophys. Res., 114, 637-644, 2009.

Yang, Y. J., Fang, Y. C., Tang, T. Y., and Ramp, S. R.: Convex and concave types of second baroclinic mode internal solitary waves, Nonlin. Processes Geophys., 17, 605-614, https://doi.org/10.5194/npg-17-605-2010, 2010.

Yuan, C., Grimshaw, R., and Johnson, E.: The evolution of second mode internal solitary waves over variable topography, J. Fluid Mech., 836, 238-259, 2018.

Zhao, Z. and Alford, M. H.: Source and propagation of internal solitary waves in the northeastern south china sea, J. Geophys. Res., 111, 63-79, 2006.

Zhao, Z., Klemas, V., Zheng, Q., and Yan, X.: Remote sensing evidence for baroclinic tide origin of internal solitary waves in the northeastern south china sea, Geophys. Res. Lett., 31, L06302, https://doi.org/10.1029/2003GL019077, 2004. 